\title{
Thyroid carcinoma with atypical metastasis to the pituitary gland and unexpected postmortal diagnosis
}

\author{
Anna Popławska-Kita1, Marta Wielogórska1, Łukasz Poplawski5, Katarzyna Siewko1, Agnieszka Adamska1,

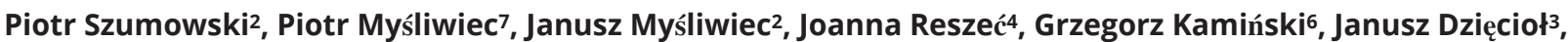 \\ Dorota Tobiaszewska1, Małgorzata Szelachowska1 and Adam Jacek Krętowski1 \\ Departments of ${ }^{1}$ Endocrinology, Diabetology and Internal Medicine, ${ }^{2}$ Nuclear Medicine, ${ }^{3}$ Human Anatomy, ${ }^{4}$ Medical \\ Pathomorphology, 5 Radiology, Medical University of Bialystok, Bialystok, Poland, ${ }^{6}$ Department of Endocrinology and \\ Radioisotopy Therapy, Military Institute of Medicine, Warsaw, Poland, and 71st Clinic Department of General and \\ Endocrine Surgery, Medical University of Bialystok, Bialystok, Poland
}

Correspondence should be addressed to A Popławska-Kita Email

annapoplawskakita@op.pl

\section{Summary}

Papillary thyroid gland carcinoma is the most common type of malignancy of the endocrine system. Metastases to the pituitary gland have been described as a complication of papillary thyroid cancer in few reported cases since 1965. We report the case of a 68-year-old female patient with a well-differentiated form of thyroid gland cancer. Despite it being the most common malignant cancer of the endocrine system, with its papillary form being one of the two most frequently diagnosed thyroid cancers, the case we present is extremely rare. Sudden cardiac arrest during ventricular fibrillation occurred during hospitalization. Autopsy of the patient revealed papillary carcinoma of the thyroid, follicular variant, with metastasis to the sella turcica, and concomitant sarcoidosis of heart, lung, and mediastinal and hilar lymph nodes. Not only does atypical metastasis make our patient's case most remarkable, but also the postmortem diagnosis of sarcoidosis makes her case particularly unusual.

\section{Learning points:}

- The goal of presenting this case is to raise awareness of the clinical heterogeneity of papillary cancer and promote early diagnosis of unexpected metastasis and coexisting diseases to improve clinical outcomes.

- Clinicians must be skeptical. They should not fall into the trap of diagnostic momentum or accept diagnostic labels at face value. Regardless of the potential mechanisms, clinicians should be aware of the possibility of the coexistence of thyroid cancer and sarcoidosis as a differential diagnosis of lymphadenopathy.

- This case highlights the importance of the diagnostic and therapeutic planning process and raises awareness of the fact that one uncommon disease could be masked by another extremely rare disorder.

\section{Background}

Differentiated thyroid cancer (DTC) is the most common endocrine malignancy with an increasing incidence of papillary thyroid cancer (PTC), which accounts for about $90 \%$ of all DTC. PTC has a strong propensity to metastasize to the cervical lymph nodes (LNs). PTC portends a favorable prognosis, even in the presence of distant metastases with a $50 \%$ rate of 5-year survival $(1,2$, 3). Pituitary metastases (PM) are found in about $1 \%$ of all pituitary resections, occurring in 1-5\% of various autopsy series (2). They often originate from the breast, lung, and gastrointestinal tract, and only $2 \%$ of these are metastases of DTC $(4,5)$. It is not clear whether these tumors involve 
the pituitary gland primarily or by secondary extension from osseous metastasis.

The published cases of metastatic thyroid cancer involving the pituitary gland were diagnosed during the investigation of symptoms and following the resection of symptomatic sellar masses $(2,3,4,5,6,7,8)$. Presenting symptoms of PM are usually some neurological signs and symptoms related to mass effect - central neurological impairment due to a space-occupying expansion in the parasellar region $(2,4)$. In contrast to pituitary adenomas, metastatic tumors, when detected, are more likely to be located in the posterior pituitary. This likely accounts for diabetes insipidus being more common in patients with PM than patients with other pituitary pathology $(3,7)$. Prognosis is poor for larger metastases. Early diagnosis is crucial and a comprehensive treatment strategy (neurosurgery, radioiodine, external radiotherapy, and radiosurgery) is recommended (8).

Coexistence of PTC and sarcoidosis may yield a diagnostic challenge as both diseases might involve the LNs. Sarcoidosis is a multisystem granulomatous disease with large variability in presentation, disease behavior, and outcome. The disease usually begins in the lungs, skin, or LNs. Less commonly affected are the eyes, liver, heart, and brain. Often, no, or only mild, symptoms are seen (9). The relationship between sarcoidosis and malignancy is not clear yet.

The goal of presenting this case is to raise awareness of the clinical heterogeneity of PTC and promote early diagnosis of unexpected metastasis and coexisting diseases to improve clinical outcomes.

\section{Case presentation}

A 68-year-old woman was in the care of an Endocrine Unit since February 2013 for the management and ongoing surveillance of multinodular goiter. An initial fine needle aspiration biopsy (FNAB) from the right lobe revealed a follicular lesion of undetermined significance (FLUS) classified as Bethesda category III. She underwent the removal of enlarged LNs (size $6 \times 2 \times 1.5 \mathrm{~cm}$ ) of the central neck in May 2013. The histopathological examination of resected tissue samples revealed nodular goiter in LNs. The patient failed to collect the results of the test and therefore was lost to follow-up.

FNAB of the thyroid was performed in January 2015 and the result was categorized as suspicious for a follicular neoplasm (FN) classified as Bethesda category IV. She underwent a total thyroidectomy in August 2015. The histopathological examination revealed nodular goiter and a fibrotic nodule within focal follicular cells in the right lobe. She received a suppressive treatment with levothyroxine after the operation.

Following surgery, the patient started suffering from neurological disturbances. She presented with headaches, non-vertiginous dizziness, double vision, and paresthesia involving half of her face. No evidence of anterior or posterior hypopituitarism was recorded. She was admitted to the Department of Neurosurgery for the investigation of these symptoms and further management.

The MRI of the pituitary gland performed in 2015 demonstrated a hypointense mass measuring approximately $17 \times 10 \times 15 \mathrm{~mm}(\mathrm{AP} \times \mathrm{LR} \times \mathrm{HF})$ and left cavernous sinus invasion (Fig. 1A). It was regarded as meningioma due to its uniform enhancement after the administration of an i.v. gadolinium contrast agent. The follow-up cranial MRI scan in July 2016 revealed a further enlargement of the pituitary mass (Fig. 1B). Anterior pituitary function tests performed preoperatively suggested a non-functioning pituitary adenoma. According to the increasing tumor size and worsening neurological symptoms, neurosurgeons qualified her for planned surgery. In January 2017, she underwent the transsphenoidal resection of the pituitary tumor. The postoperative period was complicated by deep vein thrombosis. The patient showed no improvement in her neurological condition. The surgery did not reduce the severity of symptoms, but stopped the progression of decreased visual acuity and oculomotor nerve palsies. The histopathological examination of resected mass confirmed pituitary adenoma. Due to the postoperative elevated concentration of prolactin $(78.82 \mathrm{ng} / \mathrm{mL})$, the patient was treated with a dopamine agonist - bromocriptine.

In May 2017, a follow-up cranial MRI scan revealed a significant residual mass, mostly in the sellar-suprasellar region (Fig. 1C). Chest CT showed enlarged LNs in the mediastinum and hilar areas (subcarinal LNs with dimension of $24 \times 14 \mathrm{~mm}$ ). Additionally, masses of $31 \times 18$ $\mathrm{mm}$ extending and invading the right jugular vein wall were described. In July 2017, the cytological examination of the thyroid bed remnant revealed FLUS classified as Bethesda category III.

\section{Investigation}

Owing to the thyroid pathology, progressive decline in vision, left-sided ptosis, and physical condition, the patient was admitted to the Department of Endocrinology in January 2018, in order to identify the exact cause of these symptoms. The verification of ancillary 


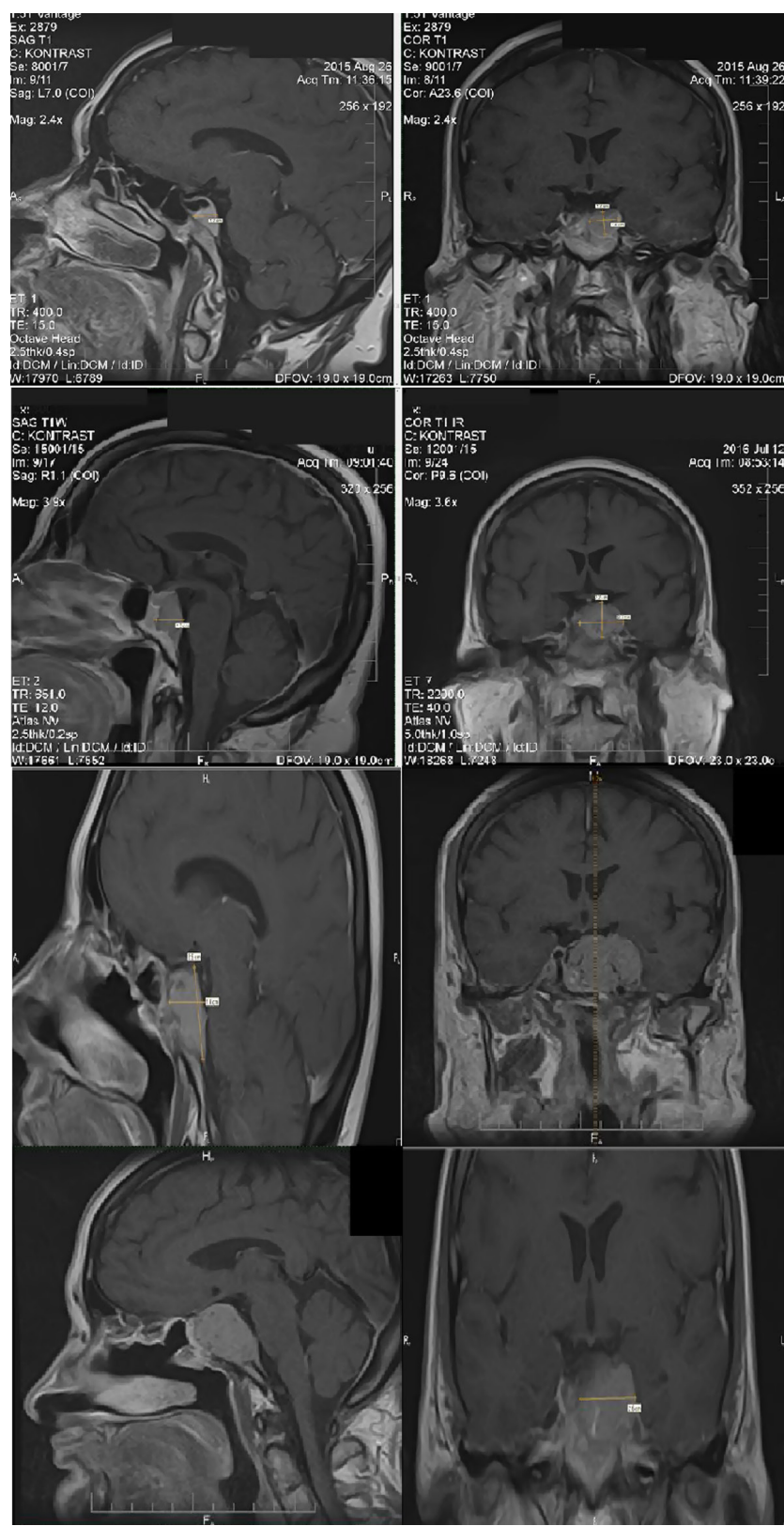

Figure 1

(A) 2015, MRI of the pituitary gland on T1-weighted image demonstrated a hypointense mass measuring approximately $17 \times 10 \times 15 \mathrm{~mm}$ $(\mathrm{AP} \times \mathrm{LR} \times \mathrm{HF})$ and left cavernous sinus invasion. It was regarded as meningioma due to its uniform enhancement after the administration of an intravenous gadolinium contrast agent; (B) 2016, MRI of the pituitary gland on T1-weighted image demonstrated a hypointense mass measuring approximately $21 \times 25 \times 28 \mathrm{~mm}(\mathrm{AP} \times \mathrm{LR} \times \mathrm{HF})$ and left cavernous sinus invasion. It was regarded as meningioma due to its uniform enhancement after the administration of an intravenous gadolinium contrast agent; (C) May 2017, MRI of the pituitary gland on T1-weighted image demonstrated a hypointense mass measuring approximately $27 \times 15 \times 25 \mathrm{~mm}$ (AP $\times \mathrm{LR} \times \mathrm{HF}$ ) mostly in the sellar and suprasellar region; (D) 2018 , MRI, the large enhancing mass $(36 \times 34 \times 24$ $\mathrm{mm}$ ) was based over the sphenoid bone with its cavernous sinus invasion, lateral displacement of infundibulum, and surrounding the right internal carotid artery without obstruction. immunohistochemical tests of the removed pituitary mass revealed the diagnosis of PM from the TC. Tumor cells stained positive for thyroglobulin and TTF-1 and were immunonegative for anterior pituitary hormones (PRL, GH, TSH, ACTH, FSH, and $\mathrm{LH}$ ) and negative for CK19, chromogranin A, and synaptophysin. The patient with the concomitant diagnoses of hypertension and type 2 diabetes was admitted to Endocrinological Department again in March 2018, with symptoms of diplopia, left complete eye ptosis, and enlargement of neck LNs. The patient was also suffering from persistent vomiting. Hormonal profile showed normal pituitary function and no evidence of diabetes insipidus. Hyponatraemia, a suspected cause of these disorders, was treated with hypertonic $\mathrm{NaCl}$ and mineralocorticoids. She underwent ultrasound examination which revealed thyroid nodule in the middle of the left lobe with irregular outline and microcalcifications and conglomerates of hypoechoic cervical masses without echogenic hilus suspicious for enlarged LNs on the right side (Fig. 2).

Chest CT performed without contrast to evaluate the extent of disease and the degree of airway obstruction revealed enlarged right cervical LNs in the II-VI region forming massive conglomeration, enlarged mediastinal LNs (paratracheal LN $22 \times 15 \mathrm{~mm}$ in diameter, subcarinal LNs $40 \times 36 \mathrm{~mm}$ in diameter) and not significantly increased in size in the left lung base, suspicious for metastases. Neither airway obstruction nor signs of interstitial lung disease were revealed. Doppler ultrasonography performed in order to evaluate blood flow through arteries and veins in the neck before operation showed hypoechoic masses along the carotid arteries without compression of the surrounding blood vessels. The right internal jugular vein was not detected. Skeletal scintigraphy did not reveal any bone metastases. Suspicions for metastases were not found in any organs except cervical, mediastinal, and pulmonary LNs.

The follow-up MRI of the sellar-suprasellar region showed further enlargement of the pituitary mass. The large enhancing mass was based over the sphenoid bone with its cavernous sinus invasion, lateral displacement of infundibulum, and surrounding the right internal carotid artery without obstruction (Fig. 1D). Fundoscopic examination did not show any signs of optic nerve atrophy.

Laboratory test results revealed hyponatraemia, normocytic anemia, decreased thyroid-stimulating hormone (TSH), follicle-stimulating hormone, and luteinizing serum level (not typical for menopausal period). Normal concentration of free thyroid hormones in 


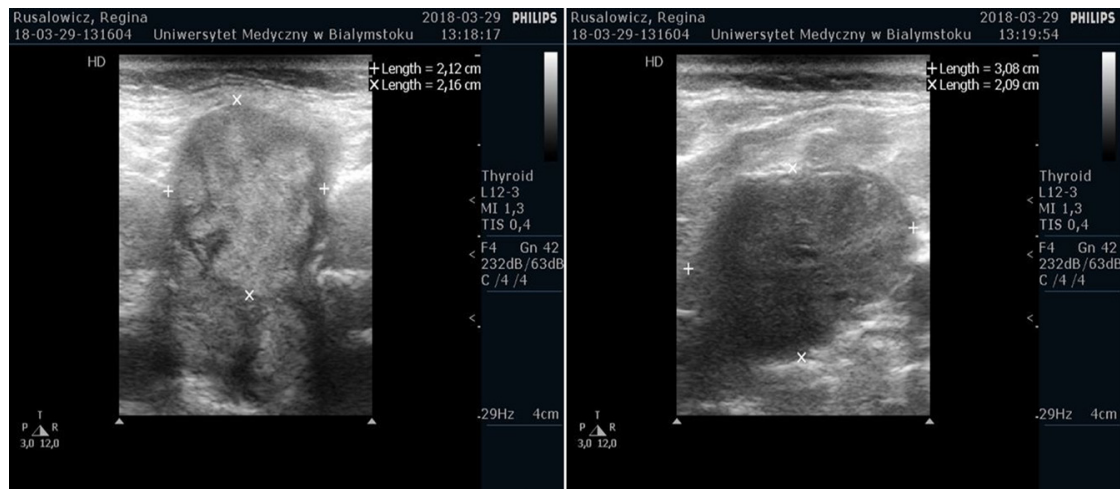

\section{Figure 2}

Neck usg. (A) The cross-section showed thyroid nodule with a diameter of $21 \times 22 \mathrm{~mm}$ in the middle of the left lobe with irregular outline; (B) showed conglomerates of hypoechoic cervical masses without echogenic hilus suspicious for enlarged LNs $(31 \times 21 \times 30 \mathrm{~mm}$ in size $)$ on the right side. addition to decreased level of TSH was considered typical for levothyroxine suppressive therapy. Post thyroidectomy thyroglobulin $(\mathrm{Tg})$ concentration was reported as higher than $500 \mathrm{ng} / \mathrm{mL}$ with the corresponding thyroglobulin antibody concentration in the normal range (Table 1).

Due to cervical LNs involvement, thyroidectomy with bilateral neck dissection was done in March 2018 . Immunohistochemical tests of the remnant and LNs were performed. Pathologist reported $18 \mathrm{LNs}$ with granulomas without necrosis and 2 of the LNs presented papillary cancer metastases. The diagnosis was made as multifocal papillary carcinoma of the thyroid gland. The examination revealed lymphadenitis with numerous well-circumscribed non-caseating granulomas within the LNs. These granulomas were composed of epithelioid histiocytes and multinucleated giant cells, macrophages, and lymphocytes without necrosis.

\section{Treatment}

Due to the diagnosed secondary adrenal insufficiency, hydrocortisone was supplemented. Owing to history of rapid increase in size of pituitary mass, the patient was qualified for a follow-up neurosurgical operation.

Table 1 Baseline laboratory results showing presence of hypopituitarism postoperatively.

\begin{tabular}{l} 
Blood investigations \\
\hline TSH, $\mu \mathrm{IU} / \mathrm{mL}$ \\
Free thyroxine, $\mathrm{ng} / \mathrm{mL}$ \\
FT3, pg/mL \\
Thyroglobulin, $\mathrm{ng} / \mathrm{mL}$ \\
ATG, IU/mL \\
ACTH, pg/mL \\
Cortisol in 24 h urine, $\mu \mathrm{g} / 24 \mathrm{~h}$ \\
Random cortisol (8am), $\mu \mathrm{g} / \mathrm{dL}$ \\
$\mathrm{PRL}, \mathrm{ng} / \mathrm{mL}$ \\
$\mathrm{FSH}, \mathrm{mIU} / \mathrm{mL}$ \\
$\mathrm{LH}, \mathrm{mIU} / \mathrm{mL}$
\end{tabular}

\begin{tabular}{cccc}
\cline { 1 - 1 } Result & & Normal reference \\
\cline { 1 - 1 } $\mathbf{0 . 1 8 9}$ & & $0.35-4.94$ \\
0.87 & & $0.7-1.48$ \\
2.50 & & $1.71-3.71$ \\
$\mathbf{7 5 0 0}$ & & $1.4-78$ \\
$\mathbf{7 . 2 9}$ & & $0-4.11$ \\
51.05 & & $7.2-63.3$ \\
6.4 & & $4.3-176$ \\
7.5 & & \\
2.12 & & $1.2-29.93$ \\
$\mathbf{3 . 8 3}$ & & $26.5-139$ \\
$\mathbf{1 . 7}$ & & $20-65$ \\
\hline
\end{tabular}

One week before the admission to the Neurosurgical Department, the patient did not consent to surgery. In view of this clinical context, she was admitted to the Endocrinological Department in May 2018. I131 wholebody scan, PET-CT scan, and echocardiography were planned to be performed. The patient was stable during admission. In the hospital, she started suffering from another episode of vomiting. Cardiac arrhythmia was not observed. Laboratory tests showed hyponatraemia, which was treated with hypertonic $\mathrm{NaCl}$. Corticosteroids were administered to avoid any potential mass effect. Due to the high probability of a blunted TSH rise after levothyroxine withdrawal postoperatively and knowing that the uptake of radioiodine into thyroidal tissue is TSH dependent, recombinant human TSH was used prior to the $\mathrm{I}^{131}$ whole-body scan (pre-therapy scan). It was decided not to give radiotherapy before RAI therapy because of the possible subsequent decrease in radioiodine uptake following tumor fibrosis that radiotherapy can cause. After two rhTSH injections $(0.9 \mathrm{mg})$ on two consecutive days, on the day of taking the ${ }^{131}$ pill, sudden cardiac arrest during ventricular fibrillation occurred. The patient died despite immediate cardiopulmonary resuscitation.

\section{Outcome and follow-up}

Autopsy of the patient revealed the follicular variant of PTC (Fig. 3A and B) with metastasis to the sella turcica (Fig. 3C) and concomitant sarcoidosis of the heart (Fig. 3H), lung, mediastinal, and hilar LNs (Fig. 3G). Immunohistochemistry of the pituitary mass confirmed the presence of thyroid cells which stained positive for thyroid transcription factor-1, cytokeratin 7 (Fig. 3D), and thyroglobulin (Fig. 3F) and negative for cytokeratin 19 (Fig. 3E) and synaptophysin. Despite the absence of CK 19 expression, a member of the keratin family responsible for the structural integrity of epithelial cells, cytological features confirmed the diagnosis. 

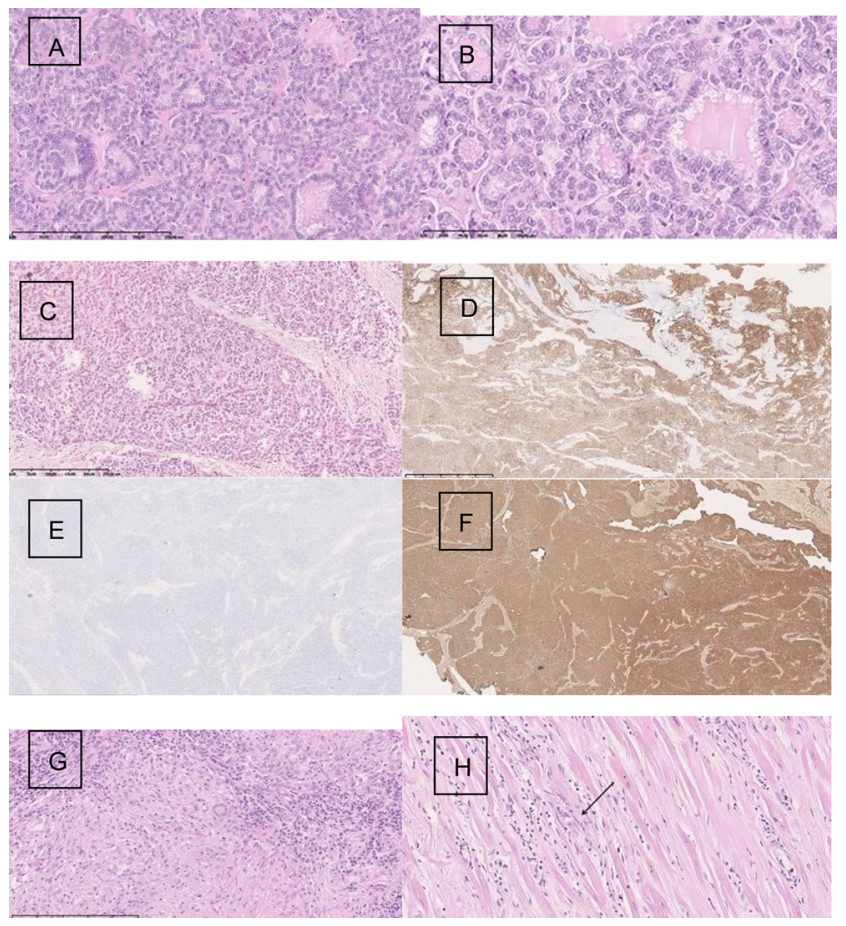

Figure 3

Histopathology of pituitary metastasis at diagnosis. (A) Thyroid gland papillary carcinoma follicular variant. Magn. 100x; (B) Papillary cancer of thyroid gland-cytological features confirm the recognition. Magn.200x; (C) Thyroid gland papillary cancer metastasis into the sellar region. Magn. 40x; (D) Cytokeratin 7 expression within the tumor cells. Magn. 40x; (E) Cytokeratin 19 negative expression within the metastase. Magn. 40x; (F) Thyreoglobulin expression within the cancer cells in metastasis. Magn. 40x; (G) Granuloma with giant cells, macrophages, and lymphocytes within the lymph node. Magn.200x; $(\mathrm{H})$ Granuloma within the cardiac muscles. Magn. 40×.

\section{Discussion}

The 2017 Bethesda System for Reporting Thyroid Cytopathology recommends subclassification of atypia of undetermined significance (AUS)/follicular lesion of undetermined significance (FLUS) into six subcategories. The initial thyroid FNAB of the thyroid nodule revealed FLUS, but the histopathological examination of the resected thyroid tissue in 2015 revealed benign changes - nodular goiter. The histopathology of enlarged LNs also revealed nodular goiter. Unfortunately, no attention was paid to the result, which revealed the presence of thyrocytes in the LNs. This finding should have raised suspicions about metastasis.

One other observation concerning our case is that the thyroid FNAB which was performed twice, in 2013 and 2015, returned false negative results for PTC. However, the final diagnosis established during the autopsy revealed the follicular variant of papillary carcinoma of the thyroid, which is partially complied with the results of FNAB categorized as FLUS or follicular neoplasm (FN).
AUS/FLUS sub-categorization helped to indicate risk for the more likely neoplasm, whether PTC or FN (10).

An important observation regarding our case is that there were no symptoms of malignancy for a number of years during the treatment period. First time malignancy was found in the pituitary adenoma. Histology of the pituitary tumor obtained in 2017 was put basing on the original clinical diagnosis (pituitary adenoma/ meningioma), elevated concentration of prolactin (78.82 $\mathrm{ng} / \mathrm{mL}$ ), associated with the histology. Small specimen was assessed, presenting monomorphic, small cells, with solid pattern, without obvious features of papillary carcinoma. There was also no information about previous thyroidectomy. The rediagnosis was done after the second reoperation of the rest of the thyroid gland due to the clinical suspicion of the thyroid cancer. PM was the first sign of the disease also in other cases. Neoplasms from almost every tissue have been reported to metastasize to the pituitary gland, with breast cancer for females and lung cancer for males accounting for approximately twothirds of all cases $(5,7,8)$. Most PM are asymptomatic, with only $7 \%$ reported to be symptomatic. Diabetes insipidus, anterior pituitary dysfunction, visual field defects, headache/pain, and ophthalmoplegia are the most commonly reported symptoms $(7,8)$. The presence of PM could be challenging to manage. Haematogenous spread can occur via the hypophyseal arteries or the portal system. As the posterior lobe of the pituitary is directly exposed to the arterial circulation, PM from other malignancies have a predilection for the posterior lobe. However, PM from thyroid carcinomas are extremely rare and seem to differ from the norm, with diabetes insipidus affecting only few of the reported cases. One suggested reason for this phenomenon is that PM from thyroid cancer tend to be relatively rapid-growing suprasellar, rather than intrasellar, lesions that destroy pituitary tissue or interrupt the pituitary stalk, hence presenting more often with mass effect $(2,3,7,8)$. Our patient presented both features, rapid growth and suprasellar expansion, but without symptoms and biochemical changes of diabetes insipidus. Radiologic evaluation was not helpful in differentiating PM from an adenoma, as no typical features of metastasis are present in CT images. Both can present with sellar enlargement and deformity with various erosions and invasions. One of the more specific MRI findings of a PM, however, is a 'dumbbell shaped' intrasellar and suprasellar tumor, with a clear indentation at the level of the diaphragma sella which has been seen in our patient since 2017 (Fig. 1C and D) (5). An adenoma usually grows slowly and can expand or destroy the 
diaphragm, whereas a PM has a rapid growth and they respect the diaphragm early in their course.

According to the reported cases, direct invasion from skull base metastasis to the pituitary gland tend to be associated with large metastases. Patients can present with symptoms related to mass effect from parasellar mass enlargement, such as ptosis, blurring of vision and oculomotor, and abducens nerve palsies, rarer symptoms include diabetes insipidus and hypopituitarism $(6,7$, $8,11,12,13)$. Our patient presented with progressive headache and blurred vision, and anterior pituitary function tests performed preoperatively suggested a nonfunctioning pituitary adenoma. Her hormonal profile revealed hypopituitarism and hyperprolactinemia from 'stalk effect,' since the transsphenoidal resection of the pituitary mass. It is highly probable that she had a direct extension from the primary ethmoid/sphenoid metastasis to the sella and then to the suprasellar region, explaining her progressive loss of vision. As this patient illustrates, PM tend to produce symptoms relating to space-occupying expansion in the parasellar region rather than to those due to the destruction of the pituitary gland.

Removal of the entire thyroid and possible metastasectomy are recommended because they will decrease the tumor loading and the amount of RAI therapy needed. If the metastases are large and locally invasive, surgical clearance may be difficult. In view of close proximity to the optic chiasm as well as cranial nerves such as the oculomotor nerve, trochlear nerve and abducens nerve, external beam radiotherapy and radiosurgery could be difficult to plan. RAI therapy is only beneficial for uptake scan positive in metastasis, as the reported uptake rate of brain metastasis ranges only from $17 \%$ to $23 \%$ (14). In patients undergoing total thyroidectomy for TC, postoperative thyroglobulin concentration accurately quantifies the risk of structural disease recurrence. Due to the dramatically increased $\mathrm{Tg}$ level in our patient, she was qualified for the RAI therapy. Sudden cardiac arrest during ventricular fibrillation occurred before I 131 therapy. The patient died almost 3 years after her initial complaint of vision disturbances and headache, which were the first symptoms of PM. Earlier diagnosis in our patient's case may have enabled performing planned tests and identifying involvement of sarcoidosis in organs. However, despite aggressive therapy, long-term survival would have been difficult to achieve. Prognosis for patients with PM is generally poor. The mean survival time is $6-7$ months, with $10 \%$ of patients dying within 1 year of diagnosis and the longest survival being 3 years (13). Autopsy of the patient revealed the follicular variant of
PTC with metastasis to the sella turcica and concomitant sarcoidosis of the heart, lung, and mediastinal and hilar LNs. Postmortem pathologist did not find lymph node with papillary cancer metastasis, instead of this all of the examined LNs presented multifocal granulomas without necrosis (19 LNs).

Coexistence of PTC and sarcoidosis may yield a diagnostic challenge as both diseases might involve the LNs of the neck. In our patient's case, sarcoidosis affected many organs but without any signs and symptoms. Enlarged LNs revealed in CT were treated as metastases from PTC.

The relationship between sarcoidosis and malignancy is not clear yet. A reduced ability to eliminate an antigen and chronic inflammation have been suggested as triggering events. Leading to a reduced tumor immune surveillance, a diminished myeloid dendritic cells function, despite upregulated co-stimulatory and maturation markers, was also raised as potential mechanism. In contrast, it has also been proposed that the occurrence of sarcoidosis is a secondary process representing a kind of systemic sarcoid reaction to a malignant tumor $(9,15,16)$.

Sarcoid reaction in the regional LNs has been reported in connection with a variety of tumors, such as PTC (16). The association of PTC and sarcoidosis with severe hypercalcaemia as the main symptom has been previously reported in the literature (17). In our patient, the postmortem diagnosis of sarcoidosis was unexpected due to a lack of pre-existing clinical evidence. LNs swelling in the case of malignancy, especially PTC, generally suggests metastases of the primary tumor. In our patient, dissection of the mass was performed for the diagnosis of metastatic PTC to the LNs, but the pathological examination revealed granuloma without caseation as well as metastasis to different LNs in March 2018 for the first time. Since the patient did not present any clinical symptoms typical of sarcoidosis, there were no suspicions of such a disease. Rizzato et al. describe peripheral LNs presentation in $11.7 \%$ of cases of sarcoidosis with no clinical evidence of changes outside (18). The finding of sarcoid-type granulomas in a peripheral lymph node without clinical evidence of changes suggestive of sarcoidosis elsewhere poses a diagnostic problem.

Cardiac involvement in sarcoidosis has been reported. It might be symptomatic or not and even revealed by sudden death. Heart conduction system is rarely investigated at autopsy, even in cases of sudden cardiac death (19). Our patient died suddenly. The histopathological examination of the heart revealed a cardiac sarcoidosis that could explain the sudden death. Cardiac sarcoidosis remains a diagnostic challenge 
and can be easily missed, clinically and pathologically. Examination of the cardiac conduction system is crucial in selected cases of sudden cardiac death. Unfortunately, 1 day before echocardiography was planned to be performed, sudden cardiac arrest during ventricular fibrillation occurred.

In conclusion, clinicians must be skeptical. They should not fall into the trap of diagnostic momentum or accept diagnostic labels at face value. Regardless of the potential mechanisms, clinicians should be aware of the possibility of the coexistence of PTC and sarcoidosis as a differential diagnosis for lymphadenopathy. This case highlights the importance of the diagnostic and therapeutic planning process and raises awareness of the fact that one uncommon disease could be masked by another extremely rare disorder.

\section{Declaration of interest}

The authors declare that there is no conflict of interest that could be perceived as prejudicing the impartiality of the research reported.

\section{Funding}

This research did not receive any specific grant from any funding agency in the public, commercial, or not-for-profit sector.

\section{Patient consent}

Written informed consent for publication of their clinical details and/ or clinical images was obtained from the patient's daughter (patient deceased).

\section{Author contribution statement}

$A \mathrm{P} \mathrm{K}, \mathrm{M} \mathrm{W}$, and $\mathrm{L} \mathrm{P}$ were involved in preparation of the initial draft and wrote the manuscript. A P K, A K, M Sz, and G K followed the patient, supervised all the reported endocrine testing, and supervised the case report preparation. L P prepared and analysed CT scans. J R performed histopathology investigations. P M performed surgical procedures. P Sz and J $M$ prepared radionuclide scans. All authors were involved in the revision and approval of the final version of the manuscript.

\section{References}

1 Haugen BR, Alexander EK, Bible KC, Doherty GM, Mandel SJ, Nikiforov YE, Pacini F, Randolph GW, Sawka AM, Schlumberger M, et al. American Thyroid Association Management Guidelines for adult patients with thyroid nodules and differentiated thyroid cancer: the American Thyroid Association Guidelines Task Force on thyroid nodules and differentiated thyroid cancer. Thyroid 201626 1-133. (https://doi.org/10.1089/thy.2015.0020)

2 Prodam F, Pagano L, Belcastro S, Golisano G, Busti A, Samà M, Caputo M, Bellone S, Voci A, Valente G, et al. Pituitary metastases from follicular thyroid carcinoma. Thyroid 201020 823-830. (https://doi.org/10.1089/thy.2009.0256)

3 Lim W, Lim DShaoting, Chng CL \& Lim AY. Thyroid carcinoma with pituitary metastases: 2 case reports and literature review. Case Reports in Endocrinology 20152015 252157. (https://doi. org/10.1155/2015/252157)

4 Madronio EB \& Lantion-Ang FL. The tale of two tumours: an undiagnosed case of papillary thyroid carcinoma. BMJ Case Reports 2011 2011 bcr0820114604. (https://doi.org/10.1136/bcr.08.2011.4604)

5 Komninos J, Vlassopoulou V, Protopapa D, Korfias S, Kontogeorgos G, Sakas DE \& Thalassinos NC. Tumors metastatic to the pituitary gland: a case report and literature review. Journal of Clinical Endocrinology and Metabolism 200489 574-580. (https://doi. org/10.1210/jc.2003-030395)

6 Vianello F, Mazzarotto R, Taccaliti A, Lora O, Basso M, Servodio O, Mian C \& Sotti G. Follicular thyroid carcinoma with metastases to the pituitary causing pituitary insufficiency. Thyroid $20112 \mathbf{2 1}$ 921-925. (https://doi.org/10.1089/thy.2010.0335)

7 Fassett DR \& Couldwell WT. Metastases to the pituitary gland. Neurosurgical Focus 200416 E8. (https://doi.org/10.3171/ foc.2004.16.2.1)

8 Barbaro D, Desogus N \& Boni G. Pituitary metastasis of thyroid cancer. Endocrine 201343 485-493. (https://doi.org/10.1007/s12020012-9806-9)

9 Kobak S, Yildiz F, Semiz H \& Orman M. Malignancy in patients with sarcoidosis. Reumatología Clínica 2019 In press. (https://doi. org/10.1016/j.reuma.2019.04.003)

10 Guleria P, Agarwal S, Iyer VK, Jain D, Mathur SR \& Yadav D. Sub categorisation of AUS/FLUS thyroid lesions as per the 2017 Bethesda System for Reporting Thyroid Cytopathology: a retrospective study from a tertiary care centre analysing risk of malignancy (ROM) of the different subcategories. Journal of Clinical Pathology 201972 771-777. (https://doi.org/10.1136/jclinpath-2019-205985)

11 Yilmazlar S, Kocaeli H \& Cordan T. Sella turcica metastases from follicular carcinoma of the thyroid. Neurological Research 200426 74-78. (https://doi.org/10.1179/016164104773026561)

12 Johnson PM \& Atkins HL. Functioning metastasis of thyroid carcinoma in the sella turcica. Journal of Clinical Endocrinology and Metabolism 1965 25 1126-1130. (https://doi.org/10.1210/jcem-25-8-1126)

13 Ntyonga-Pono MP, Thomopoulos P \& Luton JP. Pituitary metastases. 3 cases. Presse Medicale 199928 1567-1571.

14 McWilliams RR, Giannini C, Hay ID, Atkinson JL, Stafford SL $\&$ Buckner JC. Management of brain metastases from thyroid carcinoma: a study of 16 pathologically confirmed cases over 25 years. Cancer 200398 356-362. (https://doi.org/10.1002/cncr.11488)

15 Reich JM, Mullooly JP \& Johnson RE. Linkage analysis of malignancy-associated sarcoidosis. Chest 1995107 605-613. (https:// doi.org/10.1378/chest.107.3.605)

16 Cohen PR \& Kurzrock R. Sarcoidosis and malignancy. Clinics in Dermatology 200725 326-333. (https://doi.org/10.1016/j. clindermatol.2007.03.010)

17 Riis MG, Juhl KS \& Bruun JM. Concomitant sarcoidosis and papillary thyroid cancer with severe hypercalcaemia as the main symptom. BMJ Case Reports 20182018 br2017-222194. (https://doi. org/10.1136/bcr-2017-222194)

18 Rizzato G \& Tinelli C. Unusual presentation of sarcoidosis. Respiration: International Review of Thoracic Diseases 200572 3-6. (https://doi.org/10.1159/000083392)

19 Jotterand M, Grabherr S, Alexandre JA \& Michaud K. Sudden cardiac death and sarcoidosis of the heart in a young patient. Cardiovascular Pathology 201728 18-20. (https://doi.org/10.1016/j. carpath.2017.01.003)

Received in final form 3 February 2020 Accepted 21 February 2020 